\title{
Therapiebedarf und -ziele beim Systemischen Lupus Erythematodes (SLE)
}

Lupus erythematodes (LE) ist eine Autoimmunkrankheit, die zu den entzündlich rheumatischen Erkrankungen und dort zu den „Kollagenosen” gehört. Es werden 2 Hauptformen unterschieden: Der Hautlupus und der systemische Lupus erythematodes (SLE). Letzterer ist die häufigste Form des Lupus erythematodes. Neben der Haut und den Gelenken können auch alle weitere Organsysteme betroffen sein. Die typische Hautrötung im Gesicht (Schmetterlingserythem) tritt nur bei weniger als der Hälfte der SLE-Betroffenen auf.

Früher sind viele Betroffene schnell an den Folgen des SLE verstorben, heute ist der systemische Lupus Erythematodes (SLE) eine chronische Erkrankung. Das führt zu neuen Herausforderungen, vor allem die akkumulierte Krankheitsaktivität als auch die dauerhafte immunsuppressive Medikation verursachen zunehmend eine irreversible Schädigung beteiligter Organe und der Gefäße - je aktiver die Erkrankung und je intensiver die Medikation, desto früher und ausgeprägter ist das Risiko für einen Schaden. Viele SLE-Patienten werden trotz existierender Alternativen nicht optimal behandelt und das therapeutische Spektrum wird nicht voll ausgeschöpft. Wir befragten Herrn Prof. Dr. med. Matthias Schneider, Direktor der Poliklinik für Rheumatologie am Universitätsklinikum Düsseldorf, Präsident der Deutschen Gesellschaft für Rheumatologie (DGRh) und Sprecher des Kuratoriums der Lupus-Stiftung zu dieser Situation. Prof. Schneider hat im Bereich Lupus erythematodes zur Entwicklung der ACR-Response-Kriterien beigetragen und engagiert sich in der Entwicklung der Eular-Recommendations zum Lupus.

? Herr Professor Schneider, wie gut ist aus Ihrer Sicht die medizinische Versorgung von SLE-Patienten in Deutschland?

Schneider: Wenn man Deutschland weltweit im Vergleich sieht, dann haben wir sicher eine sehr gute medizinische

Versorgung auch für unsere SLE-Patientinnen (etwa 90\% der Erkrankten sind meist junge Frauen) und -Patienten. Im Vergleich zum SLE bei Schwarzen und Hispanics ist die Erkrankung in der deutschen Bevölkerung eher milde ausgeprägt, wir sehen zum Beispiel nicht so viele rasche Organversagen.
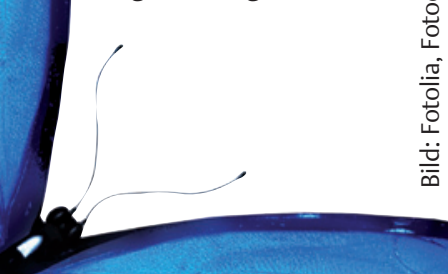

Etwas größere Schwierigkeiten haben wir in Deutschland sicher mit dem sog. Offlabel-use, dem Einsatz von zugelassenen Medikamenten in nicht zugelassener Indikation. Solche Medikamente werden etwa für ein Viertel aller Lupus-Patientinnen und -Patienten in Deutschland eingesetzt.

Wo sehen Sie bei SLE-Patienten den größten Handlungs- und Behandlungsbedarf?

Schneider: Natürlich ist vieles optimierbar in der medizinischen Versorgung von SLE-Patientinnen und Patienten. Die wichtigsten Beispiele für mich:

1. Der SLE ist in Deutschland nicht gut genug bekannt, sowohl bei Ärzten als auch bei Laien, dadurch wird er oft zu spät erkannt und behandelt.

2. Kortison wird oft, meist aus Unsicherheit unkritisch eingesetzt, weil SLE-Patienten es immer und für immer bräuchten - die Diagnose SLE ohne Aktivität ist jedoch keine Indikation für Kortison. SLE-Erkrankte gehören deshalb in die Behandlung von Spezialzentren.

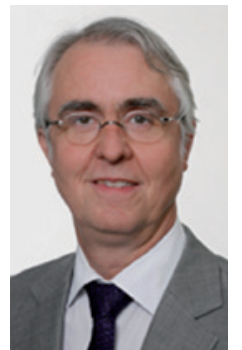

Prof. Dr. med. Matthias Schneider

3. Antimalariamittel werden zu selten eingesetzt - eigentlich sind sie das „Aspirin“ für die SLE-Patienten - sollten also immer eingesetzt werden, wenn keine Kontraindikation besteht.

4. Auch wenn die Erkrankung sehr komplex ist, sollten die Patientinnen und Patienten so gut geschult sein, dass sie ihre Erkrankung im Alltag weitgehend selbstständig händeln können.

? Welches sind für Sie die wichtigsten Therapieziele bei der SLE-Behandlung? Schneider: Das Therapieziel ist Remission, das heißt keine aktive Erkrankung unter möglichst wenig Therapie. Das soll die Patienten vor den oben angesprochenen dauerhaften Schäden schützen und ihnen ein Leben mit möglichst wenigen Einschränkungen ermöglichen.

\section{Welche Rolle spielt dabei die} Lebensqualität der Patienten? Schneider: Die Lebensqualität der Patientinnen und Patienten ist der Standard, an dem sich alle Konzepte zum SLE messen lassen müssen. Dabei besteht aktuell (noch) die große Schwierigkeit, wie wir die ausgeprägte Müdigkeit richtig einordnen und angehen können.

? Wie verändern moderne Biologika Ihren Therapiealltag - und müssen Rheumatologen eventuell lernen, umzudenken?

Schneider: Wir denken gerne um, wenn es unseren Patientinnen und Patienten hilft. Das haben wir ja bei anderen entzündlich rheumatischen Erkrankungen gezeigt. Beim SLE scheint das wie immer alles etwas komplexer zu sein. Wir sind glücklich, eine neue Substanz zugelassen zu haben. Momentan besteht unsere Aufgabe darin zu erkennen, wie wir diese Substanz möglichst optimal zum Nutzen unserer SLE-Patientinnen und Patienten einsetzen. 